\title{
Monkey Prefrontal Cortical Pyramidal and Putative Interneurons Exhibit Differential Patterns of Activity Between Prosaccade and Antisaccade Tasks
}

\author{
Kevin Johnston, ${ }^{1}$ Joseph F. X. DeSouza, ${ }^{1}$ and Stefan Everling ${ }^{1,2}$ \\ ${ }^{1}$ Department of Physiology and Pharmacology, University of Western Ontario, London, Ontario N6A 5C1, Canada, and ${ }^{2}$ Robarts Research Institute, \\ London, Ontario N6A 5K8, Canada
}

Previous studies have shown that prefrontal cortex (PFC) neurons carry task-related activity; however, it is largely unknown how this selectivity is implemented in PFC microcircuitry. Here, we exploited known differences in extracellular action potential waveforms, and antidromic identification, to classify PFC neurons as putative pyramidal or interneurons, and investigate their relative contributions to task-selectivity. We recorded the activity of prefrontal neurons while monkeys performed a blocked pro/antisaccade task in which they were required to look either toward or away from a peripheral visual stimulus. We found systematic differences in activity between neuron classes. Putative pyramidal neurons had higher stimulus-related activity on antisaccade trials, whereas putative interneurons exhibited greater activity for prosaccades. These findings suggest that task-selectivity in the PFC may be shaped by interactions between these neuronal classes. They are also consistent with the robust deficits in antisaccade performance frequently observed in disease states associated with PFC dysfunction.

\section{Introduction}

The ability to perform varied actions in response to identical sensory inputs is the hallmark of voluntary, goal-directed behavior. This ability is thought to depend on the prefrontal cortex (PFC), a neocortical area that receives input from all sensory systems and projects to motor-related cortical and subcortical areas (Fuster, 1991; Miller and Cohen, 2001). PFC lesions impair functions generally dependent on the implementation of behavioral rules, such as learning of arbitrary stimulus-response associations, and task switching (Parker and Gaffan, 1998; Bussey et al., 2001; Rushworth et al., 2005; Mansouri et al., 2007). In nonhuman primates, single PFC neurons have been shown to exhibit activity consistent with the instantiation of task rules. Responses to identical stimuli vary depending on the task and response requirements (Sakagami and Niki, 1994; Rainer et al., 1998; White and Wise, 1999; Asaad et al., 2000; Wallis et al., 2001; Everling et al., 2002; Johnston and Everling, 2006a). Although a large body of literature has demonstrated task-related activity in single PFC neurons, it is unknown how this activity is implemented within PFC microcircuitry.

The two major classes of cortical neurons are glutamatergic

Received Dec. 15, 2008; revised Feb. 18, 2009; accepted March 23, 2009.

This work was supported by grants from the Canadian Institutes of Health Research.

Correspondence should be addressed to Dr. Stefan Everling, The Centre for Brain and Mind, Robarts Research Institute, 100 Perth Drive, London, 0N N6A 5K8, Canada. E-mail: severlin@uwo.ca.

K. Johnston's present address: Centre for Neuroscience Studies, Department of Physiology, Queen's University, Kingston, 0N K7L 3N6, Canada.

J. F. X. DeSouza's present address: Centre for Vision Research, Department of Psychology, York University, Toronto, 0N M3J 13P, Canada.

DOI:10.1523/JNEUROSCI.5953-08.2009

Copyright $\odot 2009$ Society for Neuroscience $\quad$ 0270-6474/09/295516-09\$15.00/0 pyramidal neurons and GABAergic interneurons (Creutzfeldt, 1993; Markram et al., 2004; Wonders and Anderson, 2006). The dynamic interaction between these neuron types is thought to be responsible for many of the tuning properties of pyramidal neurons, and thus cortical function (Sillito et al., 1985; Wilson et al., 1994; Rao et al., 2000). Indeed, dysfunction of cortical interneurons has been implicated in disease states such as schizophrenia (Lewis et al., 2005). Electrophysiological studies have exploited known differences between the duration of the extracellularly recorded action potential waveforms of inter and pyramidal neurons to separate these two classes (Mountcastle et al., 1969; Connors and Gutnick, 1990; Markram et al., 2004). In the PFC, such studies in monkeys have implicated interneurons in tuning of spatial memory fields (Wilson et al., 1994; Rao et al., 1999, 2000; Constantinidis and Goldman-Rakic, 2002; Wang et al., 2004) and abstract numerical categories (Diester and Nieder, 2008). Such an approach has also been successful in somatosensory (Mountcastle et al., 1969; Simons, 1978; McCormick et al., 1985), motor (Merchant et al., 2008), and visual cortex (Gur et al., 1999; Mitchell et al., 2007).

Recently, we reported that PFC neurons showed task selectivity in a paradigm in which monkeys were required to alternate between blocks of prosaccade trials, in which they were required to generate a saccade to a flashed visual stimulus, and antisaccade trials (Hallett, 1978; Munoz and Everling, 2004), in which they were required to generate a saccade to the mirror location (Everling and DeSouza, 2005; Johnston and Everling, 2006b; Johnston et al., 2007). Here, we sought to investigate and quantify differences in task selectivity between cortical interneurons and pyramidal neurons. We separated putative inhibitory interneurons from putative pyramidal neurons based on their extracellular 

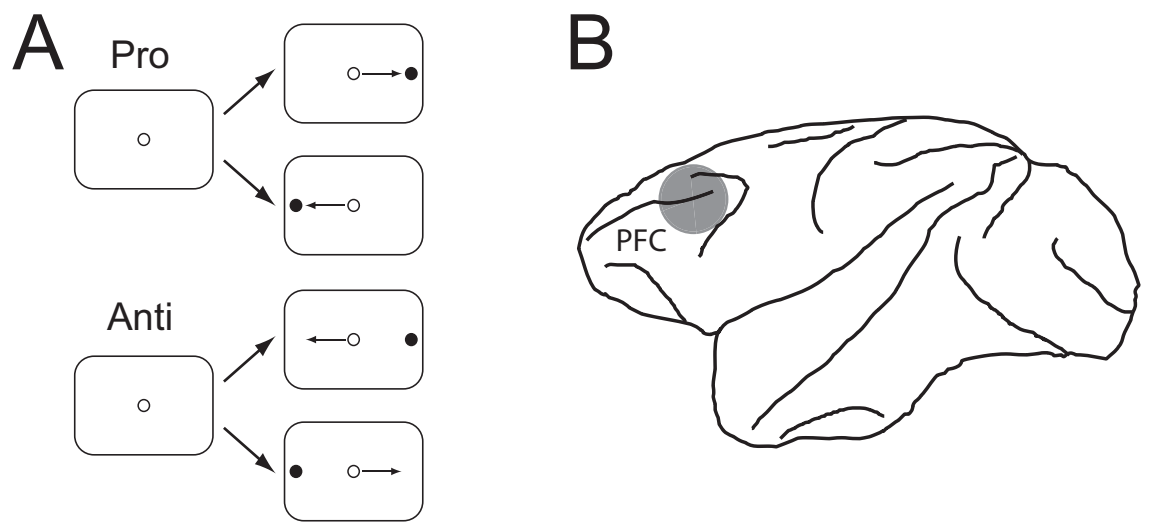

Figure 1. Task and recording area. $\boldsymbol{A}$, Schematic diagram of the behavioral task. Monkeys were required to fixate a white fixation dot at the center of the screen for $1100-1400 \mathrm{~ms}$. A peripheral stimulus then appeared to the left or right of fixation. Animals received a liquid reward if they looked toward the stimulus on prosaccade trials and to the mirror location on antisaccade trials. $B$, Location of the PFC.

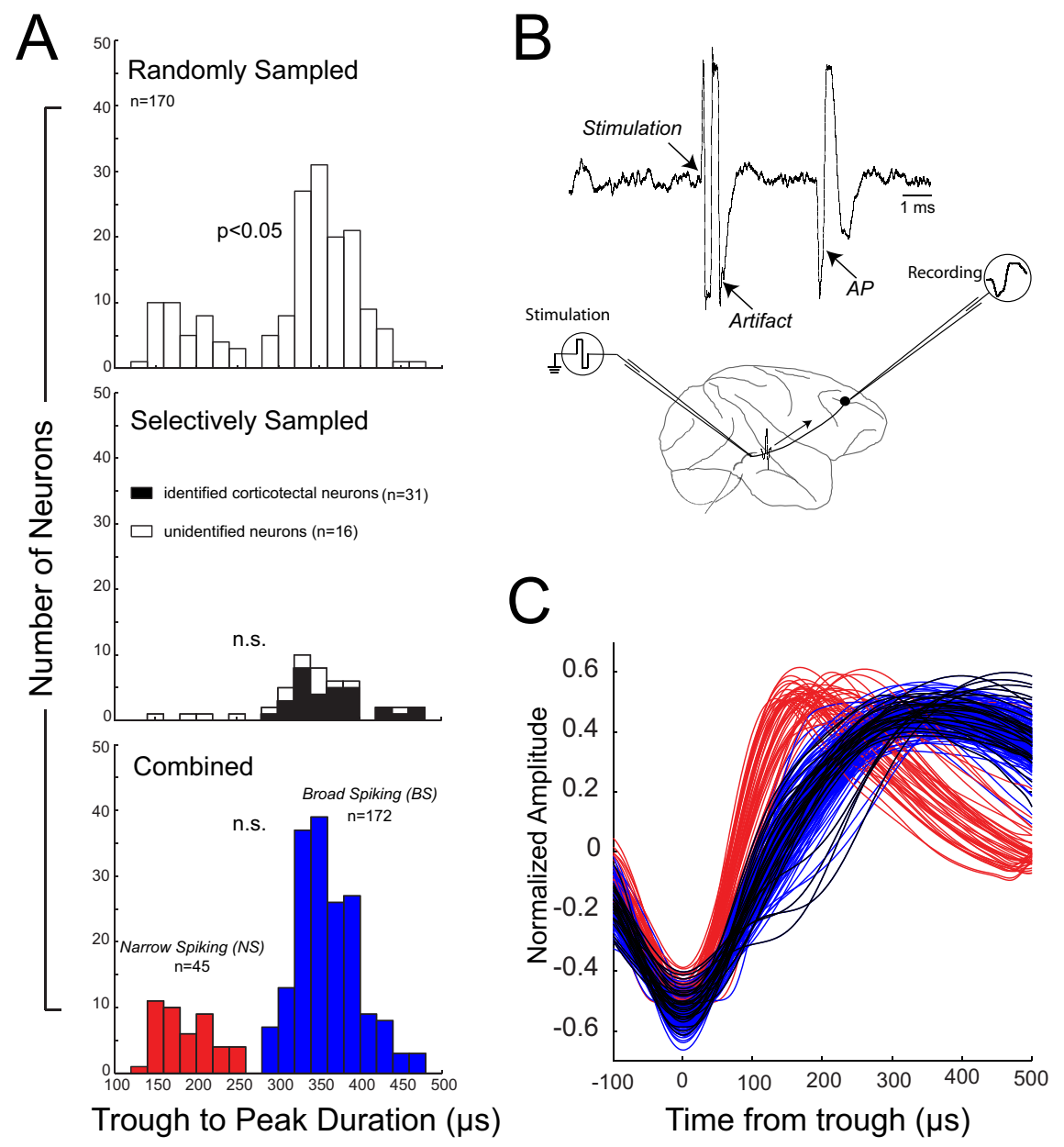

Figure 2. Classification of $B S$ and NS neurons. A, Distribution of waveform durations of randomly sampled neurons (top), selectively sampled neurons (middle), and combined recordings (bottom). Waveform duration was defined a time from trough to peak. $\boldsymbol{B}$, Left, Schematic representation of experimental method for antidromic activation of corticotectal neurons in the PFC. Right, Activation waveform showing activity recorded in the PFC. C, Normalized average waveforms of 217 neurons aligned by their trough. Red and blue correspond to narrow- and broad-spiking neurons, respectively. Waveforms of identified corticotectal neurons are plotted in black. Note that these waveforms overlap completely with other BS waveforms.

action potential durations. Furthermore, we directly identified a subset of broad spiking neurons as pyramidal using antidromic identification (Johnston and Everling, 2006b). Putative interneu- rons were more active on prosaccade trials whereas putative pyramidal neurons preferred antisaccade trials. This finding suggests that PFC task selectivity is shaped at least in part, by the interaction between these neuron classes.

\section{Materials and Methods}

All methods described (DeSouza and Everling, 2004) were performed in accordance with the guidelines of the Canadian Council of Animal Care Policy on the Use of Laboratory Animals and a protocol approved by the Animal Use Subcommittee of the University of Western Ontario Council on Animal Care. Four male rhesus monkeys (Macaca mulatta), weighing between 4 and $9 \mathrm{~kg}$, were subjects in the present experiment. Animals were prepared for chronic experiments by undergoing a surgery in which a head restraint and recording chambers were stereotaxically implanted. All four animals were implanted with recording chambers over the lateral PFC (left hemisphere in two animals, right hemisphere in one, bilateral in one; anteriorposterior: $31 \mathrm{~mm}$, medio-lateral: $18 \mathrm{~mm}$ ) such that the principal sulcus and the surrounding cortex were readily accessible. Two of the animals were also implanted with a second recording chamber, which was centered on the midline and tilted $38^{\circ}$ posterior of vertical to allow recordings from neurons in the superior colliculus (SC). Animals received analgesics and antibiotics postoperatively and were closely monitored by the university veterinarian.

\section{Behavioral task}

Each trial began with the presentation of a small fixation point at the center of the screen (Fig. $1 \mathrm{~A}$ ). Monkeys were required to fixate it within a $0.5^{\circ} \times 0.5^{\circ}$ window for a random period of $1100-1400 \mathrm{~ms}$. A visual stimulus $\left(0.15^{\circ}\right)$ was then presented pseudorandomly with equal probability $8^{\circ}$ to the left or $8^{\circ}$ to the right of the fixation spot. The central fixation point remained illuminated throughout the trial (overlap task). To receive a reward, monkeys had to generate a saccade within $500 \mathrm{~ms}$ to the stimulus on prosaccade trials or away from it to its mirror location on antisaccade trials $\left(5^{\circ} \times 5^{\circ}\right)$. After 30 correct responses, the task switched (e.g., from prosaccades to antisaccades) without any explicit signal to the monkeys [see Everling and DeSouza (2005) for details]. Eye movements were recorded at $1000 \mathrm{~Hz}$ using a magnetic search coil technique (David Northmore) in two of the animals, and a video eye tracker (ISCAN) at $120 \mathrm{~Hz}$ in the other two subjects. Monkeys performed between 4 and 20 task-switches (median 12) of alternating blocks of prosaccade and antisaccade trials.

\section{Recording method}

We recorded extracellular single neuron activity from the lateral prefrontal cortex (Fig. $1 B$ ). The locations of the implanted recording chambers were visualized in situ by MRI. Arrays of 2-8 dura-puncturing microelectrodes (FHC) were driven individually within a recording chamber by either custom-designed screw minimicrodrives that were attached to a delrin grid inside the recording 
chamber or a computer-controlled multimicroelectrode drive (NAN, Plexon). To ensure a relatively unbiased sampling of neural activity, neurons were not prescreened for taskrelated responses. Instead, we advanced the electrodes until the activity of one or more neurons was well isolated, after which data collection commenced. Waveforms were digitized, stored and sorted off-line using principal component analysis in two and three dimensions (Plexon). Waveforms were sampled at $40 \mathrm{kHz}$ (25 $\mu$ s/sample), and an 800-1000 $\mu$ s trace of each waveform was stored.

In some sessions, we used antidromic activation to identify PFC pyramidal neurons sending direct projections to the superior colliculus. Details of this procedure have been described previously (Johnston and Everling, 2006b, 2008). Briefly, arrays of three to four microelectrodes were chronically implanted in the intermediate layers of the superior colliculus ipsilateral to the PFC recording hemisphere for a period of 4-6 weeks. The activity of isolated PFC neurons was monitored while single biphasic current pulses $(0.15-0.3 \mathrm{~ms}$ per phase) were delivered to the SC through one of the implanted electrodes and an indifferent tungsten rod in the recording chamber. Neurons were required to meet several criteria including fixed latency, frequency, and collision testing, to be classified as antidromic (Lipski, 1981).

Data analysis

Data analysis was performed using customdesigned software running in Matlab (Mathworks). Trials associated with incorrect responses, broken, incorrect, or inaccurate fixation, or failure to generate a saccade within $500 \mathrm{~ms}$ were excluded from analyses of neural activity.

Waveform analysis. For each neuron, we calculated its mean extracellular waveform duration using the identical procedure to that used by Mitchell et al. (2007). For each recorded neuron, all action potentials were aligned by their troughs and averaged. This average waveform was then normalized and spline interpolated to give a resolution of $1 \mu \mathrm{s}$. Waveform duration was determined by measuring the time from the trough (negative deflection) to the peak (positive deflection). All classifications of neurons as narrow-spiking (NS) or broad-spiking (BS) were based on these values.

ANOVA. Task selective neurons were defined based on a two by two ANOVA on neural activity in the interval 100-200 ms after stimulus onset, evaluated at $p<0.05$. The factors were Task (prosaccades or antisaccades) and Stimulus Location (ipsilateral or contralateral to recorded hemisphere). Because task selectivity could be superimposed on any stimulus location preference shown by PFC neurons, neurons showing a significant main effect of Task, main effects of both Task and Stimulus Location, or a Task $\times$ Stimulus Location interaction were classified as task selective. An interaction between Task and Stimulus location would indicate that effects of stimulus location differed depending on the task being performed.

Task preference. As a measure of task preference (prosaccade vs antisaccade) in the preferred location, we computed a task preference index (TPI), as follows: TPI $=(P-A) /(P+A)$, where $P$ is activity on prosaccade trials at the preferred location in the interval 100-200 ms after stimulus onset, and $A$ is activity on antisaccade trials at the preferred location in the same interval. Positive values of this index indicate that the neuron preferred prosaccades, whereas negative values indicate the neuron preferred antisaccades.
B Ipsilateral

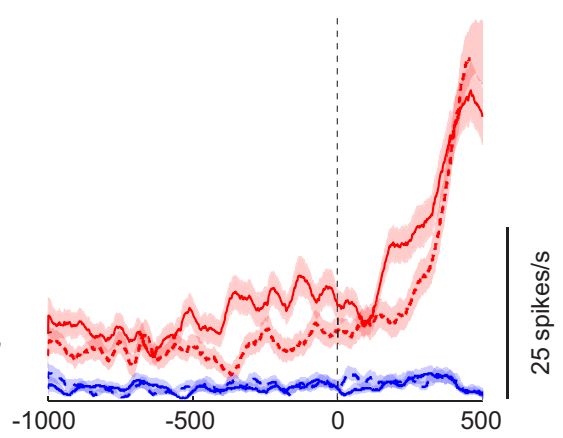

Time from Stimulus Onset (ms)

Figure 3. Activity of a single narrow- (red) and broad-spiking (blue) neuron recorded simultaneously at the tip of the same

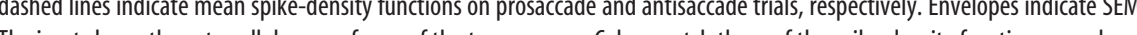
inset shows the extracellular waveforms of the two neurons. Colors match those of the spike-density functions, envelopes represent SD. $\boldsymbol{B}$, Same as $\boldsymbol{A}$, but for trials on which the stimulus appeared on the ipsilateral side.
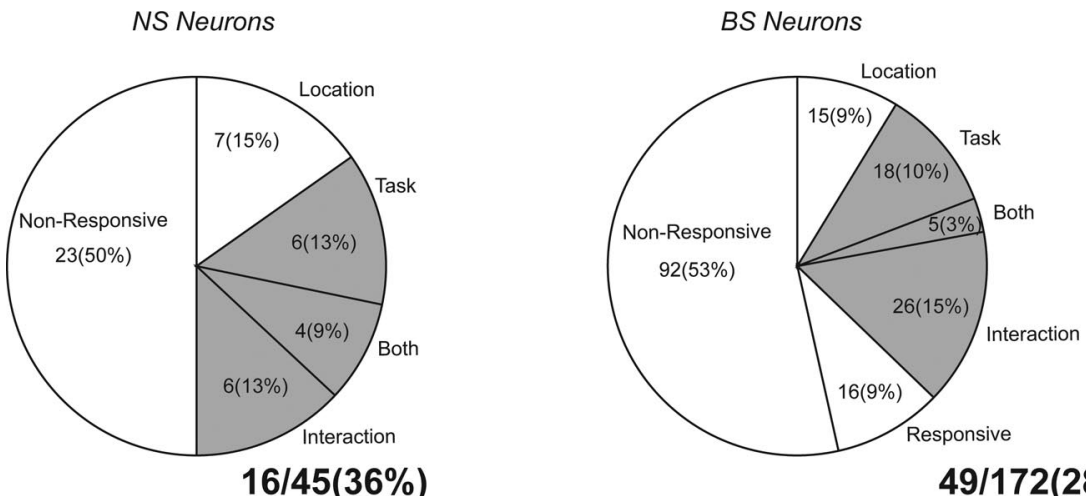

$49 / 172(28 \%)$

NS Neuron
4. Results of two-way ANOVAs on the activity in the epoch from 100 to $200 \mathrm{~ms}$ after stimulus onset for BS and NS selectivity subjected to further analysis.

Time course of task selectivity. To determine the time course of task selectivity for BS and NS neurons after stimulus presentation, we performed a sliding receiver operating characteristic (ROC) analysis. Starting from the time of peripheral stimulus presentation, the ROC value was calculated for a $50 \mathrm{~ms}$ epoch (centered around the time point). This analysis was repeated in $1 \mathrm{~ms}$ increments until $200 \mathrm{~ms}$ after stimulus presentation. An ROC time course was calculated for each neuron and then averaged separately across all BS and NS neurons with task selectivity. Values $>0.5$ indicate that the population was more active for prosaccades than antisaccades during the epoch, whereas values smaller than 0.5 indicate the population was more active for antisaccades. To test whether the ROC values were significant at any time points for the populations of NS and BS neurons, we conducted bootstrap analyses. To this end, the following procedure was repeated 10,000 times: For each neuron, a random decision was made to either exchange the two task conditions (pro and anti) (50\% probability) or leave them unchanged $(50 \%$ probability). Each of the 10,000 repetitions of the analysis, performed on all NS and BS neurons with task selectivity, yielded a single average time course. The distribution of the 10,000 average ROC values at each point in time was then used to calculate the 95 th and 5 th percentile values. Both were plotted together with the average ROC time course of the nonrandomized data. The 95 th and 5 th percentile indicate the $5 \%$ significance criterion.

Spike density function. To evaluate the relationship between neural activity and stimulus onset, continuous spike density functions were 


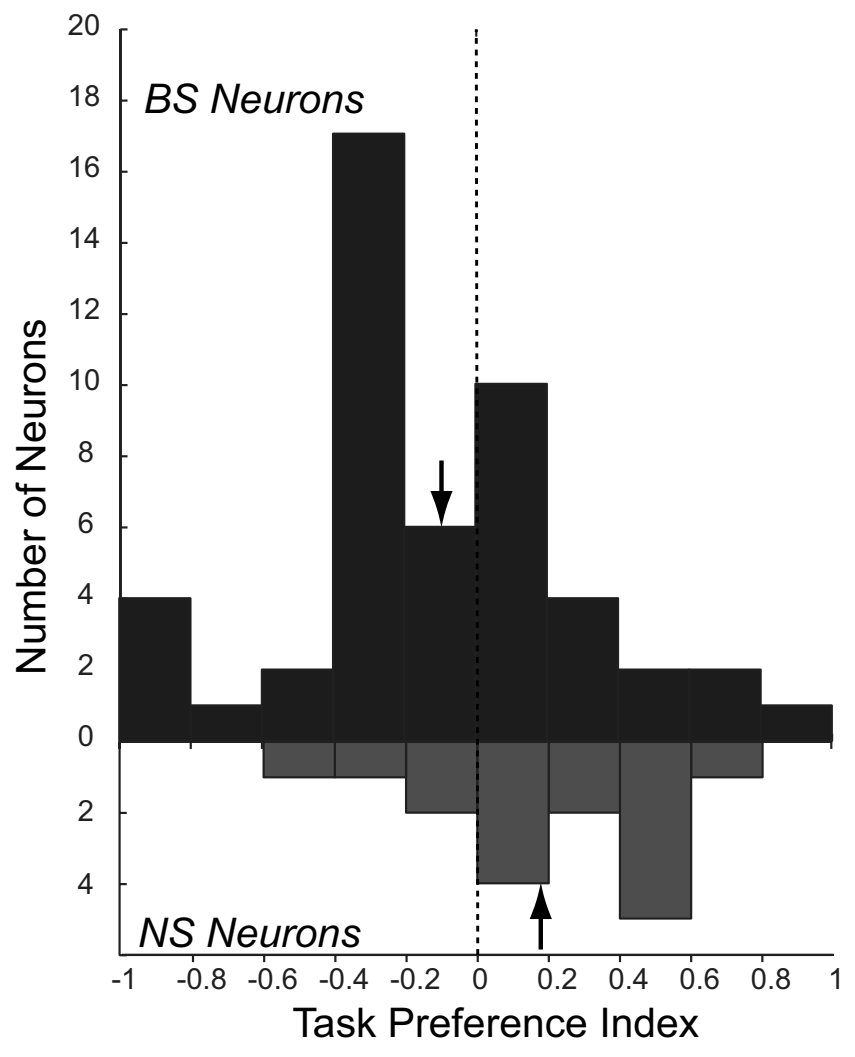

Figure 5. Values of task preference index for narrow- and broad-spiking neurons showing significant task effects in ANOVA. Negative values indicate higher activity on antisaccade trials, positive values, and prosaccade trials. Arrows indicate mean values of the index for each population of neurons.

constructed. The activation waveform was obtained by convolving each spike with an asymmetric function that resembled a postsynaptic potential (Hanes and Schall, 1996; Thompson et al., 1996; Everling et al., 1999). The advantage of this function over a standard Gaussian function (Richmond and Optican, 1987) is that a spike only exerts an effect forward and not backward in time.

\section{Results}

We recorded the extracellular waveforms of a total of 217 neurons in the lateral prefrontal cortex of four rhesus monkeys (33 in monkey K, 62 in monkey P, 80 in monkey R, 42 in monkey W) while they performed alternating blocks of prosaccades and antisaccades. Of these neurons, 170 were randomly sampled, i.e., we recorded from every neuron that we could isolate (monkeys $\mathrm{K}, \mathrm{P}$, $\mathrm{R})$. Waveform durations as defined as the time from the initial trough to peak ranged from 133 to $473 \mu$ s. Consistent with previous reports (Constantinidis and Goldman-Rakic, 2002; Mitchell et al., 2007; Diester and Nieder, 2008), we found two populations of waveforms. A histogram of waveform durations with a binwidth of $20 \mathrm{~ms}$ showed a clear bimodality (Fig, $2 \mathrm{~A}$, top) which was confirmed by a Hartigan's Dip test $(p<0.05)$ (Hartigan and Hartigan, 1985).

Previous studies have classified neurons with narrow (shorter duration) action potentials as interneurons and those with broad (longer duration) action potentials as pyramidal neurons (Constantinidis and Goldman-Rakic, 2002; Mitchell et al., 2007; Diester and Nieder, 2008). This classification was based on the known differences in waveform duration of recorded action potentials from morphologically identified interneurons and pyramidal neurons (McCormick et al., 1985; Connors and Gutnick,
1990; Nowak et al., 2003; González-Burgos et al., 2005). Such an approach is not feasible for recordings in the awake primate. As a further validation for a classification scheme based on waveform duration, we used antidromic identification techniques to identify PFC neurons sending direct projections to the SC, a midbrain oculomotor structure (Fig. $2 \mathrm{~B}$ ). These projection neurons are known to be layer V pyramidal neurons (Leichnetz et al., 1981; Creutzfeldt, 1993). We recorded a total of 47 neurons from monkeys $\mathrm{R}$ and $\mathrm{W}$ while we searched for corticotectal neurons using antidromic stimulation (Fig. $2 \mathrm{~A}$, middle). Of these 47 neurons, 31 were identified as corticotectal neurons. These identified pyramidal cells had broad extracellular waveform durations, ranging from 287 to $464 \mu \mathrm{s}$ (median $355 \mu \mathrm{s}$ ). The 16 unidentified neurons that were recorded in the same sessions had waveform durations from 180 to $453 \mu$ s (median $343 \mu$ s). The waveforms that were recorded during these selective recording sessions did not show a significant bimodality [Hartigan's Dip test $(p>$ $0.70)]$. For the remainder of the analysis, we pooled the neurons that were randomly and selectively sampled (Fig. $2 \mathrm{~A}$, bottom). The distribution of all waveforms combined showed a clear separation between NS and BS waveform durations, with no waveforms falling between 260 and $280 \mu$ s. However, a test of bimodality failed to reach significance [Hartigan's Dip test $(p=0.13)]$. We suspect that this may be because of an oversampling of pyramidal neurons, a result of the fact these neurons are easier to record because of their larger size, and because we searched selectively for this neuronal class in the sessions in which we used antidromic identification.

Based on the finding that the shortest waveform duration of identified pyramidal neurons was $287 \mu$ s, we classified neurons with waveform durations shorter than this value as NS and those with waveform durations of $270 \mu$ s or longer as BS (Fig. 2A, bottom, $C$ ).

Figure 3 shows an example of a single BS (blue) and NS neuron (red) that were recorded simultaneously from the tip of the same microelectrode while monkey $\mathrm{K}$ performed alternating blocks of prosaccades (solid lines) and antisaccades (dashed lines). Both neurons had some tonic activity before the stimulus was presented, and both neurons showed a clear spatial preference. In this case, the neurons were more active for stimuli presented on the contralateral side (Fig. 3A) compared with the ispilateral side (Fig. $3 B$ ). For stimulus presentations at this preferred location, both neurons displayed a phasic increase in discharge on prosaccade and antisaccade trials. Despite these similarities in activity, the BS and NS neurons also showed a number of clear differences in their activity pattern. First, the NS neuron had higher levels of both tonic and phasic activity than the BS neuron. Higher activity levels for putative interneurons than putative pyramidal neurons have been previously reported by extracellular recording studies in nonhuman primates (Mitchell et al., 2007; Diester and Nieder, 2008). In fact, the higher discharge rates of NS neurons have been used as an additional classification tool (Constantinidis and Goldman-Rakic, 2002). Second, the NS neuron also exhibited an increase in discharge for the ipsilateral location. The BS neuron did not show any increase in activity at the ipsilateral location. Third, the discharge rate of the NS neuron in response to presentation of the peripheral stimulus was higher on prosaccade trials, whereas the BS neuron was more active on antisaccade trials.

To identify NS and BS neurons that showed task-related responses, we performed ANOVAs on the poststimulus activity of each neuron (see Materials and Methods). We found that similar proportions of NS and BS neurons exhibited task-related activity 
(Fig. 4). In the sample of 45 NS neurons, we found that 16 neurons (36\%) showed task selective activity. In the sample of 172 BS neurons, 49 neurons (28\%) exhibited task selective activity. In addition to neurons that exhibited some form of task selectivity, we also found NS and BS neurons that were location-selective but not task selective (15\% of NS neurons and $9 \%$ of BS neurons). Some of the BS neurons were responsive, i.e., they changed their activity in the interval from 100 to $200 \mathrm{~ms}$ after stimulus presentation compared with the $200 \mathrm{~ms}$ period immediately before stimulus onset (tested with paired $t$ tests), but they were not task- or location-selective ( $9 \%$ of BS).

To compare task-related activity between NS and BS neurons, we computed task preference indexes for all task selective NS and BS neurons (see Materials and Methods). The distribution of task preference indices is plotted in Figure 5. The mean $( \pm$ SEM) task preference index of BS neurons was $-0.11 \pm 0.05$, indicating that the population of BS neurons was more active on antisaccade than on prosaccade trials. In contrast, NS neurons were more active on prosaccade than on antisaccade trials (mean task preference index $=$ $0.19 \pm 0.08)$. Task preferences differed significantly between BS and NS neurons $(p<0.01$, Mann-Whitney $U$ test $)$.

Next, we compared the activity of BS and NS neurons with task selectivity on prosaccade and antisaccade trials for trials on which the stimulus was presented on the side contralateral to the recorded hemisphere, and those on which it was presented on the ipsilateral side. We found recently that electrical microstimulation has clear contralateral effects on task performance on prosaccade and antisaccade trials (Wegener et al., 2008). Figure 6A shows the activity of the population of BS (red lines) and NS (blue lines) neurons for prosaccade (solid lines) and antisaccade trials (dashed lines) at the contralateral location. NS neurons exhibited a stimulusrelated response on prosaccade trials that was attenuated on antisaccade trials. BS neurons had a larger stimulus-related response on antisaccade than prosaccade trials. We compared the activity of NS and BS neurons between prosaccade and antisaccade trials in an early stimulus period (100-150 ms after stimulus onset) and a late stimulus period (150-200 ms after stimulus onset) as a coarse measure of the relative timing of selectivity between these neuronal classes. The activity of the population of NS neurons was significantly different between prosaccade and antisaccade trials in the early, but not late period (Fig. 6B), whereas BS neurons exhibited significant differences in both early and late periods (Fig. 6C). For trials on which the stimulus was presented on the ipsilateral side (Fig. 6D), NS neurons exhibited a marked increase in stimulus-related activity that was significant during the early stimulus period (Fig. 6 E). BS neurons hemisphere.
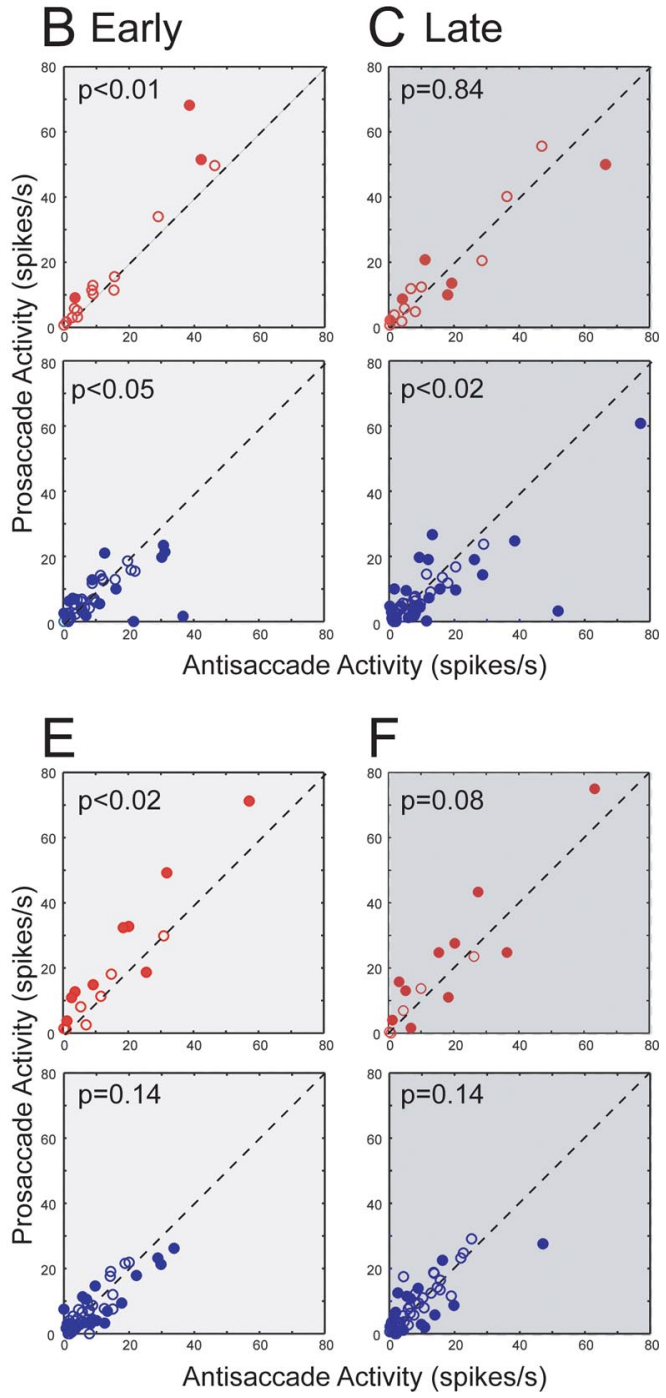

Figure 6. Activity in the selected neuron samples for prosaccade and antisaccade trials. A, Mean spike density of narrowspiking (red) and broad-spiking (blue) neurons on prosaccade (solid lines) and antisaccade trials (dashed lines) for trials on which the stimulus appeared contralateral to the recording hemisphere. $\boldsymbol{B}$, Activities of individual narrow-spiking (red) and broadspike counts in the epoch from 100 to $150 \mathrm{~ms}$ after stimulus onset. Filled circles indicate neurons with significant differences ( $p<$ 0.05 , Wilcoxon signed rank test) between prosaccade and antisaccade trials. $\boldsymbol{C}$, Same as $\boldsymbol{B}$, but for activities in the epoch from 150 to $200 \mathrm{~ms}$ after stimulus onset. $\boldsymbol{D}-\boldsymbol{F}$, Same as $\boldsymbol{A}-\boldsymbol{C}$, but for trials on which the stimulus appeared ipsilateral to the recording

showed only a very small increase in discharge rate and no differences between prosaccade and antisaccade trials (Fig. $6 F$ ). These findings demonstrate that both NS and BS neurons display task selectivity but that there are clear differences in the nature of selectivity across these neuron classes. First, NS neurons were more active on prosaccade trials, whereas BS neurons were more active on antisaccade trials. Second, differences in laterality of task selectivity between NS and BS neurons were observed, such that the preference of NS neurons for prosaccade trials was present for both contralateral and ipsilateral stimulus presentations, whereas the preference of BS neurons for antisaccade trials was significant only for contralateral stimulus presentations. Third, we observed coarse differences in the timing of task selectivity between classes. Selectivity appeared in the early, but not late, epoch in the population of NS neurons, and within both early and late epochs in the population of BS neurons.

To perform a more principled comparison of the relative time 

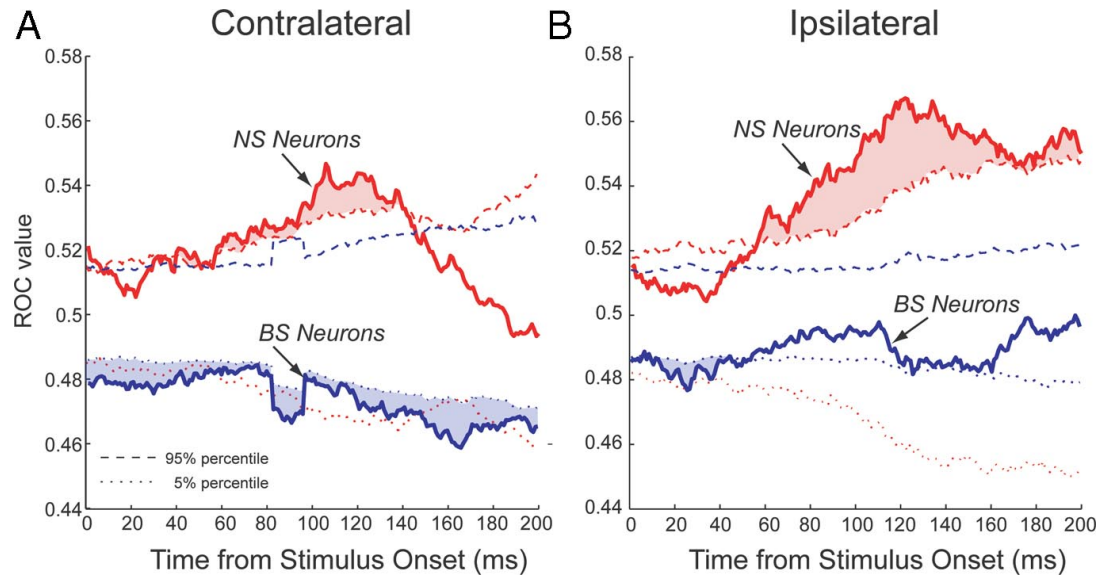

Figure 7. Time course of task selectivity of narrow- and broad-spiking neurons. $\boldsymbol{A}$, Time course of average population ROC values (solid lines) of NS (red) and BS (blue) PFC neurons on trials on which the stimulus appeared contralateral to the recording hemisphere. Dashed and dotted lines indicate 95th and 5th percentile of randomized data, respectively. Red lines represent percentile values for narrow-spiking neurons, blue, percentile values for broad-spiking neurons. Periods in which the solid lines lay above the dashed line or below the dotted line (red and blue shaded regions) indicate periods with significant task selectivity ( $p<0.05$ ). $\boldsymbol{B}$, Same as $\boldsymbol{A}$, but for trials on which the stimulus appeared on the ipsilateral side.

courses of task selectivity after stimulus onset for NS and BS neurons, we performed an ROC analysis. To test whether these ROC values were significantly different from chance, we also conducted bootstrap analyses (see Materials and Methods). For contralateral stimulus presentations, BS neurons exhibited task selectivity earlier than NS neurons (Fig. 7A). For ipsilateral stimulus presentations, BS neurons showed some early task selectivity, whereas NS neurons displayed later and more robust task selective responses (Fig. 7B). Examination of Figure 7 also shows that NS neurons were more active on prosaccade trials ( ROC values $>0.5$ ), whereas BS neurons were more active on antisaccade trials ( $\mathrm{ROC}$ values $<0.5$ ). This finding verifies that task preferences were opposite between neuron classes, and indicates that BS neurons displayed task selectivity earlier than NS neurons.

\section{Discussion}

Task selective responses are a ubiquitous property of PFC neurons, but little is known about how such selectivity is implemented in PFC microcircuitry. Here, we used known differences in the extracellular waveforms of cortical pyramidal and interneurons as a classification tool (Constantinidis and GoldmanRakic, 2002; González-Burgos et al., 2005; Mitchell et al., 2007; Diester and Nieder, 2008), and compared the response properties of narrow-spiking putative interneurons (NS), and broadspiking putative pyramidal neurons (BS) in a blocked pro/antisaccade task. We found systematic differences in the response properties of these two neuronal types suggestive of different functional roles in performance of the task. First, NS neurons exhibited a higher level of activity on prosaccade than antisaccade trials, whereas BS neurons showed the opposite pattern of selectivity. Second, task selectivity of BS neurons was lateralized, whereas that of NS neurons was present for both contralateral and ipsilateral stimulus presentations. Third, task selectivity evolved earlier in BS than NS neurons. Together, these data suggest that task selectivity of prefrontal pyramidal neurons may be shaped, in part, by the activity of inhibitory interneurons.

\section{The broad-spiking class as pyramidal neurons}

Consistent with several previous studies (Constantinidis and Goldman-Rakic, 2002; González-Burgos et al., 2005; Mitchell et al., 2007; Diester and Nieder, 2008), we found that the distribution of extracellular waveform durations exhibited a clear bimodality. NS neurons also had higher levels of activity than BS neurons. Previous studies have argued that NS waveforms originate from the spiking of interneurons, whereas BS waveforms have been attributed to pyramidal neurons. The main argument in support of this classification has been the temporal profile of intracellular recorded activity of interneurons and pyramidal neurons and the differences in ion-channel kinetics between these types of neurons (Martina et al., 1998; González-Burgos et al., 2004; Bean, 2007). These differences have been confirmed by direct comparisons between in vitro intracellular and extracellular recordings of action potentials in the monkey dorsolateral PFC (González-Burgos et al., 2005).

Here, we used antidromic stimulation to directly identify layer $\mathrm{V}$ pyramidal neurons that project to the superior colliculus. We found the waveform durations of PFC neurons recorded here fell into two distinct and clearly separated populations, and that all identified output neurons had trough to peak durations longer than $270 \mu \mathrm{s}$, placing them within the BS class. Although this result is based on a fairly small sample size ( 31 neurons), this finding provides further support for the assumption that BS neurons are mainly pyramidal neurons whereas NS neurons are interneurons. Our finding is also consistent with a previous report that identified corticotectal neurons in the primate frontal eye field as BS neurons (Shin and Sommer, 2006).

\section{Task-related differences in prefrontal cortex}

Neural correlates of behavioral rules and categories have been found in many studies of PFC function (Rainer et al., 1998; White and Wise, 1999). Miller and Cohen (2001) proposed a model in which PFC neurons participate in cognitive control by actively maintaining patterns of activity representing task requirements and sending outputs that modulate the activity of other brain areas in the service of behavioral goals. The need for such control is especially high in situations when strong direct stimulusresponse mappings must be overridden in favor of weaker, purposive ones (Miller, 1999; Miller and Cohen, 2001). The antisaccade task (Hallett, 1978) represents a clear example of a task with such requirements. Subjects are instructed not to look toward a flashed visual stimulus but instead to look to the opposite side. As such, the antisaccade task has become a popular tool for the study the flexible behavioral control (Everling and Fischer, 1998; Munoz and Everling, 2004). Patients with prefrontal damage (Guitton et al., 1985; Pierrot-Deseilligny et al., 1991), and psychiatric disorders such as schizophrenia (Fukushima et al., 1988) reliably make performance errors in this task consistent with an inability to inhibit reflexive saccades toward the visual stimulus.

Task-related differences between prosaccade and antisaccades have been found in PFC neurons (Funahashi et al., 1993; Everling and DeSouza, 2005; Johnston and Everling, 2006a; Johnston et al., 2007), as well as a number of other cortical and subcortical areas including the frontal eye field (FEF) (Everling and Munoz, 2000), anterior cingulate cortex (ACC) (Johnston et al., 2007), supplementary eye field (SEF) (Schlag-Rey et al., 1997; Amador et 

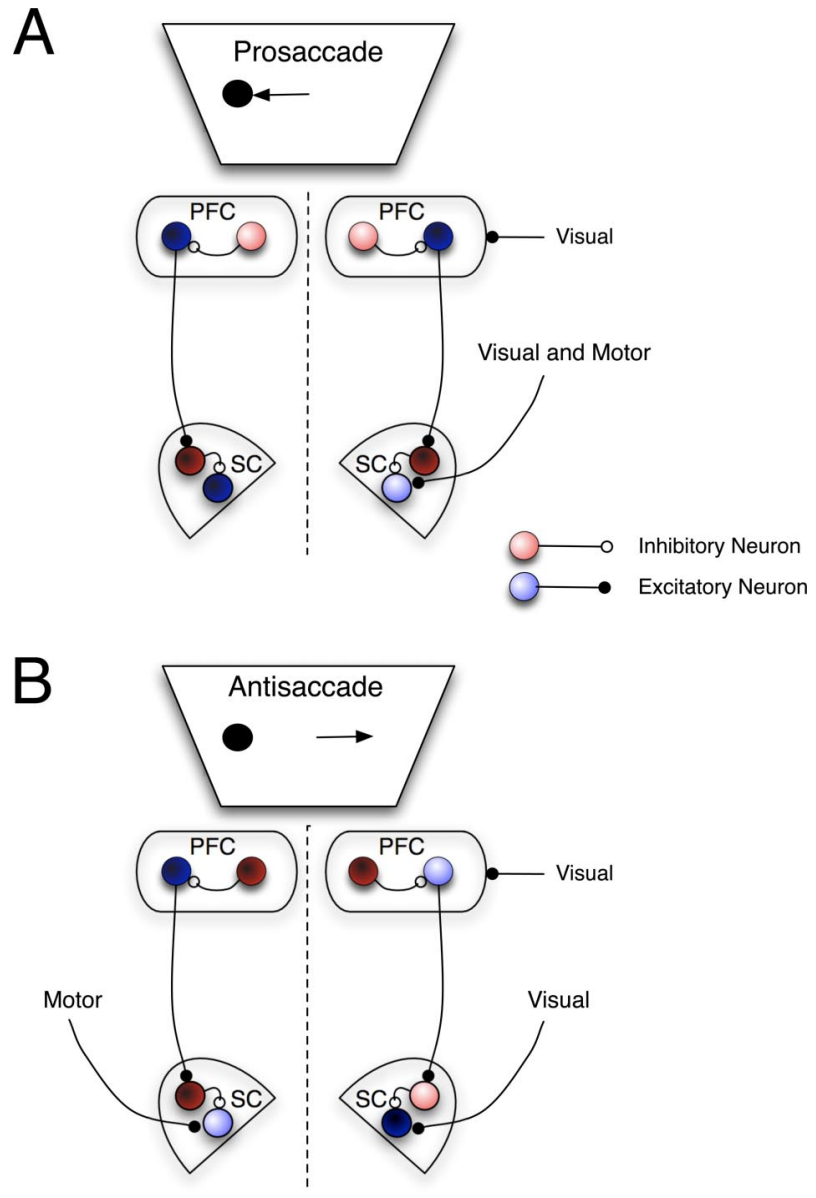

Figure 8. Schematic representation of putative mechanism underlying contribution of BS and NS neurons to antisaccade and prosaccade task performance. Blue circles represent excitatory neurons, red circles, inhibitory neurons. Color saturation represents activity level, lighter: higher activity, darker: lower activity. A, Prosaccade trial on which visual stimulus appears in left hemifield and correct response is a leftward saccade. Inhibitory NS neurons in PFC are more active on prosaccade trials and reduce activity of BS PFC output neurons to SC. This leads to reduced activity of SC fixation or inhibitory interneurons (dark red circles) and reduced inhibition of $\mathrm{SC}$ saccade neurons. Visual input from stimulus in left hemifield and motor command for leftward saccade can combine to drive activity of saccade neuron and generate correct leftward prosaccade. Enhanced activity of inhibitory NS neurons could, therefore, attenuate outputs of BS neurons andallow reflexive prosaccade to proceed. $\boldsymbol{B}$, Antisaccadetrial on which visual stimulus appears in left hemifield, and a rightward saccade (arrow) is the correct response. Inhibitory intermeurons in PFC (dark red circles) are less active on antisaccade trials and thus allow the following chain of events to proceed. Visual stimulus evokes responses in excitatory, BS output neurons (light blue circle) of the right PFC and saccade neuron (dark blue circle) of the right SC. Excitatory input of BS neuron in right PFC increases activity of inhibitoryinterneuron or fixation neuron in SC (pinkcircle), which attenuates stimulus-driven visual activity ofSCsaccadeneuron and preventsitfrom reaching threshold for generating asaccade. Thus, reflexive saccades toward the visual stimulus areinhibited. Voluntarymotor inputthen drives saccadeneuron in leftSC (light blue circle) to generate correctrightward saccade. Decreased activity of inhibitory NS neurons in PFC on antisaccade trials could therefore act to allow outputs of $\mathrm{BS}$ neurons to regulate SC activity in support of antisaccade performance.

al., 2004), lateral intraparietal area (LIP) (Gottlieb and Goldberg, 1999; Zhang and Barash, 2000), globus pallidus (Yoshida and Tanaka, 2008), and SC (Everling et al., 1998, 1999). Based mainly on differences in activity of SC neurons during prosaccade and antisaccade tasks, Munoz and Everling proposed an accumulator model in which correct antisaccade performance depends on top-down control of SC saccade neurons by frontal cortical areas (Munoz and Everling, 2004). Direct support for this hypothesis is provided by the finding that corticotectal PFC neurons are more active on antisaccade than prosaccade trials (Johnston and Everling, 2006b). The current study indicates that not only output neurons but also interneurons in the PFC carry task-related activity.

Implications for representation of task selectivity in PFC

Previous models of the interactions between pyramidal neurons and inhibitory interneurons (Compte et al., 2000; Wang et al., 2004; Fusi et al., 2007) have made basic assumptions that are in partial agreement with our data. Such models assume that the activity of inhibitory interneurons is driven by pyramidal cell input, is broadly tuned, and is not task selective. The first two assumptions are consistent with the earlier onset of task selectivity we observed in BS than NS neurons, and the broad tuning we observed in NS neurons. Here, however, we observed robust task selective responses in NS neurons. This, in combination with the numerosity-selective responses recently observed in NS neurons (Diester and Nieder, 2008), suggests that inhibitory interneurons may play a more active role in the implementation of top-down control than previously thought.

Our finding that task selectivity appeared earlier in the population of BS than NS neurons is contrary to the latency differences between these neuron classes in some previous studies. Both Wilson et al. (1994) and Diester and Nieder (2008) found that putative interneurons had shorter visual response latencies than putative pyramidal neurons. This discrepancy may be the result of differences between the tasks they used and the pro/antisaccade task used here. Differences in preparatory activity between prosaccade and antisaccade trials has been observed in many areas, and is thought to reflect a presetting of the oculomotor circuitry in advance of stimulus presentation to facilitate suppression of reflexive saccades (Munoz and Everling, 2004). We have previously reported that preparatory activity of corticotectal PFC neurons is greater on antisaccade than prosaccade trials (Johnston and Everling, 2006b). We speculate that the early task selectivity we observed in BS neurons could reflect changes in preparatory set that were not present in the saccade and delayed match to sample tasks used in other studies.

We have previously reported that lateralized increases of the activity of corticotectal PFC neurons on antisaccade trials represents a suppression signal that acts to decrease the activity of SC saccade neurons and thereby prevent the saccade signal from reaching threshold (Munoz and Everling, 2004; Johnston and Everling, 2006b). This could be mediated via an enhancement of the activity of either fixation or inhibitory interneurons in the SC. A schematic depiction of this model is presented in Figure 8. For example, on an antisaccade trial on which the visual stimulus appears to the left of fixation (Fig. 8A), output (BS) neurons in the right PFC would show an enhancement of activity. Since cortical outputs are excitatory (Creutzfeldt, 1993), this leads to increased activity of either fixation or interneurons in the right SC, and a concomitant reduction in the activity of SC saccade neurons, suppressing a reflexive saccade toward the stimulus. On prosaccade trials (Fig. $8 \mathrm{~B}$ ), this enhanced signal is absent, and visual inputs are allowed to drive SC saccade neurons. This would facilitate performance on prosaccade trials, since the visual stimulus location and saccade direction are the same. By such a mechanism, cortical output selective for the antisaccade "rule" could act to modulate the downstream SC to facilitate the antisaccade "goal." Here, we found that NS neurons have a reduced stimulusrelated response on antisaccade trials. We hypothesize that as a consequence, pyramidal neurons receive less inhibition during the stimulus period on antisaccade trials and therefore increase their response to contralateral stimulus presentations. Although the limitations of extracellular recording in the awake animal 
preclude us from making direct empirical statements about connectivity between the NS and BS neurons recorded here, our results, combined with the demonstrated anatomical connectivity between interneurons and pyramidal neurons, suggest that interaction between task selective responses of these neuronal types could be one mechanism that acts to regulate cortical output and thus performance in the antisaccade task.

Our results suggest that the decrease of activity of interneurons in the PFC immediately after stimulus presentation might be a crucial step in correct antisaccade performance. Dysfunction of some classes of cortical inhibitory interneurons has been demonstrated in schizophrenic patients (Lewis et al., 2005). Hence, a disruption in the normal function activity of PFC interneurons could contribute to the well established performance deficits of schizophrenic patients in the antisaccade task (Fukushima et al., 1988; Broerse et al., 2001; Hutton and Ettinger, 2006; Gooding and Basso, 2008).

\section{References}

Amador N, Schlag-Rey M, Schlag J (2004) Primate antisaccade. II. Supplementary eye field neuronal activity predicts correct performance. J Neurophysiol 91:1672-1689.

Asaad WF, Rainer G, Miller EK (2000) Task-specific neural activity in the primate prefrontal cortex. J Neurophysiol 84:451-459.

Bean BP (2007) The action potential in mammalian central neurons. Nat Rev Neurosci 8:451-465.

Broerse A, Crawford TJ, den Boer JA (2001) Parsing cognition in schizophrenia using saccadic eye movements: a selective overview. Neuropsychologia 39:742-756.

Bussey TJ, Wise SP, Murray EA (2001) The role of ventral and orbital prefrontal cortex in conditional visuomotor learning and strategy use in rhesus monkeys (Macaca mulatta). Behav Neurosci 115:971-982.

Compte A, Brunel N, Goldman-Rakic PS, Wang XJ (2000) Synaptic mechanisms and network dynamics underlying spatial working memory in a cortical network model. Cereb Cortex 10:910-923.

Connors BW, Gutnick MJ (1990) Intrinsic firing patterns of diverse neocortical neurons. Trends Neurosci 13:99-104.

Constantinidis C, Goldman-Rakic PS (2002) Correlated discharges among putative pyramidal neurons and interneurons in the primate prefrontal cortex. J Neurophysiol 88:3487-3497.

Creutzfeldt OD (1993) Cortex cerebri. Goettingen: Springer.

DeSouza JF, Everling S (2004) Focused attention modulates visual responses in the primate prefrontal cortex. J Neurophysiol 91:855-862.

Diester I, Nieder A (2008) Complementary contributions of prefrontal neuron classes in abstract numerical categorization. J Neurosci 28:7737-7747.

Everling S, DeSouza JF (2005) Rule-dependent activity for prosaccades and antisaccades in the primate prefrontal cortex. J Cogn Neurosci 17:1483-1496.

Everling S, Fischer B (1998) The antisaccade: a review of basic research and clinical studies. Neuropsychologia 36:885-899.

Everling S, Munoz DP (2000) Neuronal correlates for preparatory set associated with pro-saccades and anti-saccades in the primate frontal eye field. J Neurosci 20:387-400.

Everling S, Dorris MC, Munoz DP (1998) Reflex suppression in the antisaccade task is dependent on prestimulus neural processes. J Neurophysiol 80:1584-1589.

Everling S, Dorris MC, Klein RM, Munoz DP (1999) Role of primate superior colliculus in preparation and execution of anti-saccades and prosaccades. J Neurosci 19:2740-2754.

Everling S, Tinsley CJ, Gaffan D, Duncan J (2002) Filtering of neural signals by focused attention in the monkey prefrontal cortex. Nat Neurosci 5:671-676.

Fukushima J, Fukushima K, Chiba T, Tanaka S, Yamashita I, Kato M (1988) Disturbances of voluntary control of saccadic eye movements in schizophrenic patients. Biol Psychiatry 23:670-677.

Funahashi S, Chafee MV, Goldman-Rakic PS (1993) Prefrontal neuronal activity in rhesus monkeys performing a delayed anti-saccade task. Nature 365:753-756.

Fusi S, Asaad WF, Miller EK, Wang XJ (2007) A neural circuit model of flexible sensorimotor mapping: learning and forgetting on multiple timescales. Neuron 54:319-333.

Fuster JM (1991) The prefrontal cortex: anatomy, physiology, and neuropsychology of the frontal lobe. New York: Raven.

González-Burgos G, Krimer LS, Urban NN, Barrionuevo G, Lewis DA (2004) Synaptic efficacy during repetitive activation of excitatory inputs in primate dorsolateral prefrontal cortex. Cereb Cortex 14:530-542.

González-Burgos G, Krimer LS, Povysheva NV, Barrionuevo G, Lewis DA (2005) Functional properties of fast spiking interneurons and their synaptic connections with pyramidal cells in primate dorsolateral prefrontal cortex. J Neurophysiol 93:942-953.

Gooding DC, Basso MA (2008) The tell-tale tasks: a review of saccadic research in psychiatric patient populations. Brain Cogn 68:371-390.

Gottlieb J, Goldberg ME (1999) Activity of neurons in the lateral intraparietal area of the monkey during an antisaccade task. Nat Neurosci 2:906-912.

Guitton D, Buchtel HA, Douglas RM (1985) Frontal lobe lesions in man cause difficulties in suppressing reflexive glances and in generating goaldirected saccades. Exp Brain Res 58:455-472.

Gur M, Beylin A, Snodderly DM (1999) Physiological properties of macaque V1 neurons are correlated with extracellular spike amplitude, duration, and polarity. J Neurophysiol 82:1451-1464.

Hallett PE (1978) Primary and secondary saccades to goals defined by instructions. Vision Res 18:1279-1296.

Hanes DP, Schall JD (1996) Neural control of voluntary movement initiation. Science 274:427-430.

Hartigan JA, Hartigan PM (1985) The dip test of unimodality. Ann Statistics 13:70-84.

Hutton SB, Ettinger U (2006) The antisaccade task as a research tool in psychopathology: a critical review. Psychophysiology 43:302-313.

Johnston K, Everling S (2006a) Neural activity in monkey prefrontal cortex is modulated by task context and behavioral instruction during delayedmatch-to-sample and conditional prosaccade-antisaccade tasks. J Cogn Neurosci 18:749-765.

Johnston K, Everling S (2006b) Monkey dorsolateral prefrontal cortex sends task-selective signals directly to the superior colliculus. J Neurosci 26:12471-12478.

Johnston K, Everling S (2008) Task-relevant output signals are sent from monkey dorsolateral prefrontal cortex to the superior colliculus during a visuospatial working memory task. J Cogn Neurosci. 21:1023-1038.

Johnston K, Levin HM, Koval MJ, Everling S (2007) Top-down controlsignal dynamics in anterior cingulate and prefrontal cortex neurons following task switching. Neuron 53:453-462.

Leichnetz GR, Spencer RF, Hardy SG, Astruc J (1981) The prefrontal corticotectal projection in the monkey; an anterograde and retrograde horseradish peroxidase study. Neuroscience 6:1023-1041.

Lewis DA, Hashimoto T, Volk DW (2005) Cortical inhibitory neurons and schizophrenia. Nat Rev Neurosci 6:312-324.

Lipski J (1981) Antidromic activation of neurones as an analytic tool in the study of the central nervous system. J Neurosci Methods 4:1-32.

Mansouri FA, Buckley MJ, Tanaka K (2007) Mnemonic function of the dorsolateral prefrontal cortex in conflict-induced behavioral adjustment. Science 318:987-990.

Markram H, Toledo-Rodriguez M, Wang Y, Gupta A, Silberberg G, Wu C (2004) Interneurons of the neocortical inhibitory system. Nat Rev Neurosci 5:793-807.

Martina M, Schultz JH, Ehmke H, Monyer H, Jonas P (1998) Functional and molecular differences between voltage-gated $\mathrm{K}+$ channels of fastspiking interneurons and pyramidal neurons of rat hippocampus. J Neurosci 18:8111-8125.

McCormick DA, Connors BW, Lighthall JW, Prince DA (1985) Comparative electrophysiology of pyramidal and sparsely spiny stellate neurons of the neocortex. J Neurophysiol 54:782-806.

Merchant H, Naselaris T, Georgopoulos AP (2008) Dynamic sculpting of directional tuning in the primate motor cortex during three-dimensional reaching. J Neurosci 28:9164-9172.

Miller EK (1999) Neurobiology. Straight from the top. Nature 401:650-651.

Miller EK, Cohen JD (2001) An integrative theory of prefrontal cortex function. Annu Rev Neurosci 24:167-202.

Mitchell JF, Sundberg KA, Reynolds JH (2007) Differential attention- 
dependent response modulation across cell classes in macaque visual area V4. Neuron 55:131-141.

Mountcastle VB, Talbot WH, Sakata H, Hyvärinen J (1969) Cortical neuronal mechanisms in flutter-vibration studied in unanesthetized monkeys. Neuronal periodicity and frequency discrimination. J Neurophysiol 32:452-484.

Munoz DP, Everling S (2004) Look away: the anti-saccade task and the voluntary control of eye movement. Nat Rev Neurosci 5:218-228.

Nowak LG, Azouz R, Sanchez-Vives MV, Gray CM, McCormick DA (2003) Electrophysiological classes of cat primary visual cortical neurons in vivo as revealed by quantitative analyses. J Neurophysiol 89:1541-1566.

Parker A, Gaffan D (1998) Memory after frontal/temporal disconnection in monkeys: conditional and non-conditional tasks, unilateral and bilateral frontal lesions. Neuropsychologia 36:259-271.

Pierrot-Deseilligny C, Rivaud S, Gaymard B, Agid Y (1991) Cortical control of reflexive visually guided saccades. Brain 114:1473-1485.

Rainer G, Asaad WF, Miller EK (1998) Selective representation of relevant information by neurons in the primate prefrontal cortex. Nature 393:577-579.

Rao SG, Williams GV, Goldman-Rakic PS (1999) Isodirectional tuning of adjacent interneurons and pyramidal cells during working memory: evidence for microcolumnar organization in PFC. J Neurophysiol 81:1903-1916.

Rao SG, Williams GV, Goldman-Rakic PS (2000) Destruction and creation of spatial tuning by disinhibition: $\mathrm{GABA}_{\mathrm{A}}$ blockade of prefrontal cortical neurons engaged by working memory. J Neurosci 20:485-494.

Richmond BJ, Optican LM (1987) Temporal encoding of two-dimensional patterns by single units in primate inferior temporal cortex. II. Quantification of response waveform. J Neurophysiol 57:147-161.

Rushworth MF, Buckley MJ, Gough PM, Alexander IH, Kyriazis D, McDonald KR, Passingham RE (2005) Attentional selection and action selection in the ventral and orbital prefrontal cortex. J Neurosci 25:11628-11636.

Sakagami M, Niki H (1994) Encoding of behavioral significance of visual stimuli by primate prefrontal neurons: relation to relevant task conditions. Exp Brain Res 97:423-436.
Schlag-Rey M, Amador N, Sanchez H, Schlag J (1997) Antisaccade performance predicted by neuronal activity in the supplementary eye field. Nature 390:398-401.

Shin SY, Sommer MA (2006) Frontal eye field input neurons have higher spontaneous firing rates and narrower action potentials than output neurons. Soc Neurosci Abstr 32:138.11.

Sillito AM, Salt TE, Kemp JA (1985) Modulatory and inhibitory processes in the visual cortex. Vision Res 25:375-381.

Simons DJ (1978) Response properties of vibrissa units in rat SI somatosensory neocortex. J Neurophysiol 41:798-820.

Thompson KG, Hanes DP, Bichot NP, Schall JD (1996) Perceptual and motor processing stages identified in the activity of macaque frontal eye field neurons during visual search. J Neurophysiol 76:4040-4055.

Wallis JD, Anderson KC, Miller EK (2001) Single neurons in prefrontal cortex encode abstract rules. Nature 411:953-956.

Wang XJ, Tegnér J, Constantinidis C, Goldman-Rakic PS (2004) Division of labor among distinct subtypes of inhibitory neurons in a cortical microcircuit of working memory. Proc Natl Acad Sci U S A 101:1368.

Wegener SP, Johnston K, Everling S (2008) Microstimulation of monkey dorsolateral prefrontal cortex impairs antisaccade performance. Exp Brain Res 190:463-473.

White IM, Wise SP (1999) Rule-dependent neuronal activity in the prefrontal cortex. Exp Brain Res 126:315-335.

Wilson FA, O'Scalaidhe SP, Goldman-Rakic PS (1994) Functional synergism between putative gamma-aminobutyrate-containing neurons and pyramidal neurons in prefrontal cortex. Proc Natl Acad Sci U S A 91:4009-4013.

Wonders CP, Anderson SA (2006) The origin and specification of cortical interneurons. Nat Rev Neurosci 7:687-696.

Yoshida A, Tanaka M (2008) Enhanced modulation of neuronal activity during antisaccades in the primate globus pallidus. Cereb Cortex 19:206-217.

Zhang M, Barash S (2000) Neuronal switching of sensorimotor transformations for antisaccades. Nature 408:971-975. 